\title{
COMMENT ON A PAPER OF C. ULUÇAY
}

\section{W. T. SCOTT}

There is a gap in the proof given in [1] that the Bloch-Landau constant satisfies $\mathfrak{A}>.629$. After some correspondence with the author of [1] it appears that a satisfactory proof of the result is not available. Reproduced below are the steps which lead to the gap.

The function $f(z)$ is univalent and satisfies

$$
|f(z)| \leqq \frac{1}{2} \log \frac{1+|z|}{1-|z|}=|z| M(|z|),|z|<1 .
$$

For each fixed $s, 0<s<1, f(z, s)=f(s z) / s$ is univalent and satisfies $|f(z, s)| \leqq M(s)$ for $|z|<1$. For $t>0, \Phi\left(z^{\prime}\right)=z^{\prime} /\left(1 \pm t z^{\prime}\right)^{2}$ is univalent for $\left|z^{\prime}\right|<1 / t$ and is not univalent (or regular) in any larger concentric disc. Also

$$
t(z, s, t)=t M(s)\left\{\Phi\left[(f(z, s) / t M(s))^{3}\right]\right\}^{1 / 3}
$$

is univalent for $|z|<1$ provided

$$
|f(z, s) / t M(s)|^{3}<1 / t, \quad|z|<1,
$$

and the univalence of $\tilde{f}(z, s, t)$ for $|z|<1$ is thus assured only if $t>1$. If $f(z)$ omits $c$ then $\tilde{f}(z, s, t)$ omits

$$
\gamma(s)=(c / s)\left[1 \pm t^{-2} c^{3} / s^{3} M^{3}(s)\right]^{-2 / 3} .
$$

If $\left|\arg c^{3}\right| \leqq \pi / 2$ and the plus sign is used in $\gamma(s)$, then when $t=t_{c}$ is so chosen that $\gamma(s)>0$, the condition

$$
\arg c^{3}-2 \arg \left[1+c^{3} / t_{c}^{2} s^{3} M^{3}(s)\right]=0
$$

must hold. By an elementary theorem of geometry, $\left|c^{3}\right| / t_{c}^{2} s^{3} M^{3}(s)=1$, and a similar argument shows that $t_{c}$ satisfies this same relation in the case where $\pi / 2 \leqq\left|\arg c^{3}\right| \leqq \pi$. The subsequent part of the proof in [1] involves the univalence of $\tilde{f}\left(z, s, t_{c}\right)$ as $s \rightarrow 1$. Because of the restriction on $t_{c}$ only those values of $s$ may be used for which $s M(s)$ $<|c|$ and, since $M(s) \rightarrow \infty$ as $s \rightarrow 1$, it is not permissible to let $s \rightarrow 1$. Added in proof. See review by E. Reich, Math. Rev. vol. 19 (1958) p. 736.

\section{REFERENCE}

1. C. Ulusay, Bloch functions of the third kind and the constant $\mathfrak{A}$, Proc. Amer. Math. Soc. vol. 8 (1957) pp. 923-925.

\section{NORTHWESTERN UNIVERSITY}

Received by the editors September 23, 1958. 